\title{
The effect of including power converter losses when modelling energy storage systems: a UK Domestic Study.
}

\author{
Konstantina Panagiotou*, Christian Klumpner, Mark Sumner \\ THE UNIVERSITY OF NOTTINGHAM \\ University Park, NG7 2RD \\ Nottingham, UK \\ Tel.: +44 (0) 1159515151 \\ Fax: +44 (0) 1159513666 \\ E-Mail: *eexkp3@,nottingham.ac.uk
}

\section{Keywords}

«Battery Management Systems (BMS) », « Energy system management », « Energy storage».

\begin{abstract}
Energy storage systems (ESS) are seen as an increasingly important technology for managing electrical distribution systems, and there is now much research into both the underlying technologies of these systems, and their "optimum" management especially for applications within a single household. Optimum management usually depends on many factors and assumptions, for example what should be optimized (self-consumption of local renewable resources, consumer cost, peak reduction), prediction of local generation and load patterns, and the assumptions concerning the operation of the ESS itself. This paper aims to quantify one of the usual assumptions made (especially by non-electrical engineers) i.e. that the assumption that the power converter in the ESS is $100 \%$ efficient does not lead to substantial errors in the ESS performance and cost-benefit analysis. Three different ESS power converter models have been created and their behaviour as part of a house based ESS management system over a one year period has been analysed using five different control approaches, a variety of battery and solar panel sizes and employing a time of use tariff (Economy 7). By observing the ESS charging pattern through the year and monitoring of the annual electricity cost along with the system's losses for the household, relationships between the battery and PV size, the control algorithms, the electricity cost and the system losses were created.
\end{abstract}

\section{Introduction}

The potential benefits of energy storage (ES) are widely recognised, from energy arbitrage through to providing frequency regulation in a power grid. What is less certain is the business case for employing ES for these different services [1]. There are many reasons for this financial uncertainty: one of them is the requirement for a standard approach to modelling and evaluating the behaviour of an energy storage system (ESS) when applied to specific applications. As there are many types of ES technologies, and they can be installed at three different levels (behind the meter, at distribution or at transmission level), it is essential to define a technology-based systems approach to modelling which can combine the necessary temporal granularity to accurately represent ES [2].

In this paper, the installation of an ESS in a UK house with an installed Photovoltaic (PV) system is considered. A domestic installation was chosen to be examined since storage often has the greatest value to the power system when it is placed closest to the source of demand rather than at the transmission or the distribution level [3]. Three different ES systems were modeled which considered different levels of system efficiency, and particular included a model of the power converter losses. In order to make the use of an ESS viable, a time of use tariff needs to be considered so that the battery can be charged at off-peak overnight rates as well as from excess PV generated locally. For this work a UK "Economy 7" pricing scheme was taken into consideration, as this is an existing tariff available in the UK. In order to investigate the impact of neglecting the power converter losses, the cost of electricity for the householder was determined for different control algorithms, battery capacities and PV sizes over a period of one year to provide recommendations for system design and sizing. 


\section{The Energy Storage System and Data Sources used for the Simulation Studies}

\section{Description of the Overall Energy Storage System}

As a real UK domestic ES installation is being examined in this study, real consumption and generation data was used for the power profile - this data will be discussed later in the paper. The house's net power profile was taken to be the generated power from the PV minus the required power for the house. If the power profile is positive, this excess energy will be either used for charging the battery or it will be exported to the power grid (at a relatively low tariff compared to import tariff). Alternatively, if the power consumption is greater than the generation, the consumed power will be either supplied by the battery (battery discharging) or by the purchased power from the grid. Most, if not all of the consumption by the household occurs in the peak tariff period. Therefore the aim of the battery controller is to shift as much energy used by the household as possible either to the overnight off peak tariff period, or to be provided by any excess PV generation.

The overall system which was simulated is illustrated in Fig.1. As it can be seen from the figure, the simulated ESS consists of a battery in series with a power converter. More specifications of the battery and converter models used can be found in the two following sections. The Economy 7 pricing scheme was used and the battery charges between 00:00 and 07:00 every day because this is the time of the off-peak electricity tariff, until it reaches a predefined state of charge (SOC) level. The overnight charging level for each day depends on the different control algorithms used. For some of the proposed algorithms access to the internet or the use of a calendar timer is necessary. More details concerning the different control algorithms and their implementation can be found later in the paper. After 07:00 and before the next day, the battery charges or discharges by following the house's net power profile until either the battery is fully charged or discharged, or until the beginning of the next day (24:00). In order to ensure that the ESS operates within its safety limits, a Battery Management System (BMS) was assumed to be installed between the power profile controller and the ESS. The BMS monitors the SOC, the current and the power in and out of the ESS, in order to disconnect it when the SOC of the battery reaches the lower limit (10\%) or upper limit $(90 \%)$ : these limits prevent the over-discharge and over-charge of the battery respectively. Additionally, the BMS ensures that the ESS will not operate at high currents and so, the charging current is controlled to be lower than C (nominal battery capacity) and the discharging current no greater than $2 \mathrm{C}$.

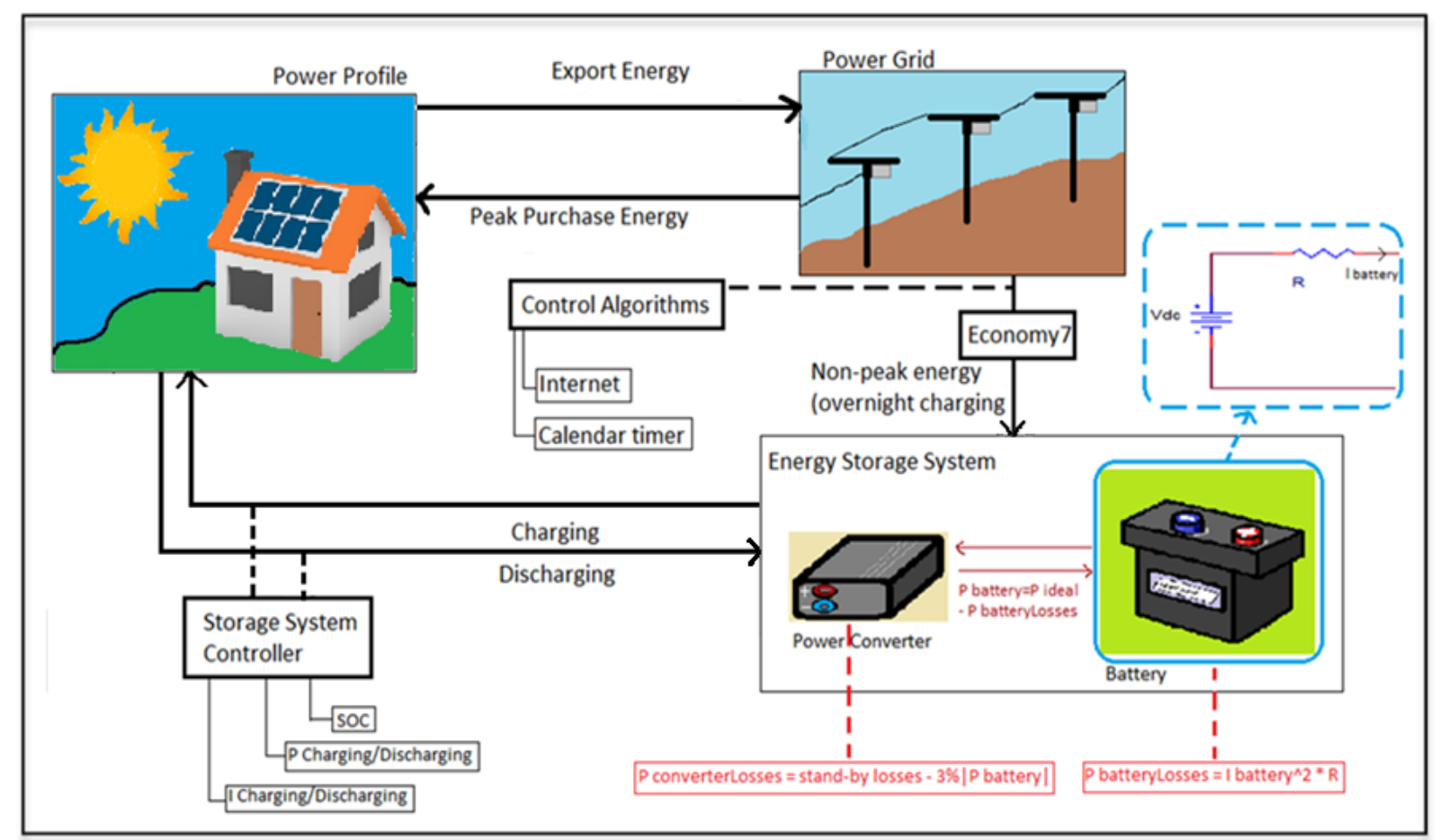

Fig. 1: Simulated Energy Storage System (ESS) 


\section{Battery Model}

One objective of this project is to create a model which can describe as much about the real behaviour of a Lead-Acid battery as possible, but be simple enough to implement as part of a real time control system to inform the battery charge/discharge pattern, without violating any SOC or current limits. Many battery models already exist in the literature. Most of them are not well suited to be combined with a performance model [4]. Electrochemical models (the most accurate) are too complex for this application, whilst mathematical models are too simple [5]. Stochastic and analytic models are equally limited. Electric-circuit models were considered to be the most suitable, as they have an average level of complexity, without compromising performance [5], [6].

As batteries are not ideal voltage sources since their open circuit voltage changes with SOC, the model with variable efficiency (1) was chosen. The parameters of a Leoch battery were taken from its datasheet for a 12V-33Ah Lead-Acid battery [7]. This battery chemistry was chosen, since it is the lowest cost choice at present and in the near future and has positive public acceptance [2], [8]. For different battery sizes, the internal resistance was adjusted by assuming that same type of batteries were connected in parallel and so by increasing the battery size, the internal resistance decreased.

$$
P \text { batteryLosses }=I \text { battery }{ }^{2} * R
$$

\section{Converter Model}

In order to represent a complete ESS, a power electronic converter was assumed to connect the battery to the AC grid. The power rating for the converter was set at $3 \mathrm{~kW}$ for this particular installation. Three different cases were examined for the converter model. The first (Converter Model 1-CM1) assumed, as seen in many economic models that the converter is considered to be ideal and has $100 \%$ efficiency, and hence no losses. For the other cases, the converter losses are modeled as in (2), consisting of two terms: a constant $\mathbf{P}_{\mathbf{s b}}=60 \mathrm{~W}$ representing the standby losses that account for the power consumed by the control platform, the gate drivers, the display, the transducers and the cooling fans, and a proportional term with the power processed by the converter that would account for a $3 \%(\mathbf{k} \%)$ of the battery power which account for semiconductors and filter losses. It is clear that as the power level decreases, the losses due to the standby loss become predominant resulting in poorer efficiency.

$$
P \text { converterLosses }=P \mathrm{sb}+k_{\%} * \mid P \text { battery } \mid
$$

Converter model 2 (CM2) assumes that there is no restriction for the ESS operation and that it always tries to deliver/absorb the battery power handled also in CM1, with the only difference now, the converter losses will force the household to buy additional energy to cover the losses. As it became clear that the efficiency degrades at lower power levels, it was recognized that a way to limit the degradation of the round trip efficiency of the ESS was to disable the operation of it when the power level is lower than a given level, which will be referred as the cut-off power. There are many ways to identify its optimum level. First approach was based on a statistical analysis that evaluates the time that the ESS spends operating at a particular power level and then quantifies the total amount of losses created by the operation of ESS down to the cut-off point. Fig. 2a shows the variation of cumulative energy loss over a week versus the choice of the cut-off power level for each of the four seasons considered. In Fig. 2b, the cumulative ESS operating time as percentage of the week is also shown versus the cut-off power level which is useful for the understanding of the results. It can be noted that when the system is allowed to operate with lower powers, the amount of energy losses increases, partly because the efficiency is poorer but more importantly because the ESS operates for significantly longer times. A change in the slope of the loss curve can be noticed for some of the seasons when the cut-off power drops below 900W (winter) and this is caused by the need to charge with more energy overnight. This part of the curve can be significantly changed by the user by allowing overnight charge with higher power over shorter time (2-3 hours rather than 7 hours). This behaviour is not seen in the summer curve as the PV provides sufficient energy and the overnight charging is minimized. If the losses need to be heavily reduced, the ESS cut-off power needs to be quite high. For example, losses can be reduced to $4 \mathrm{kWh}$ from more than $12 \mathrm{kWh}$, if the cut-off power is limited to $500 \mathrm{~W}$ but this will result also to an operating time of only $1 / 4$ of the week time. 

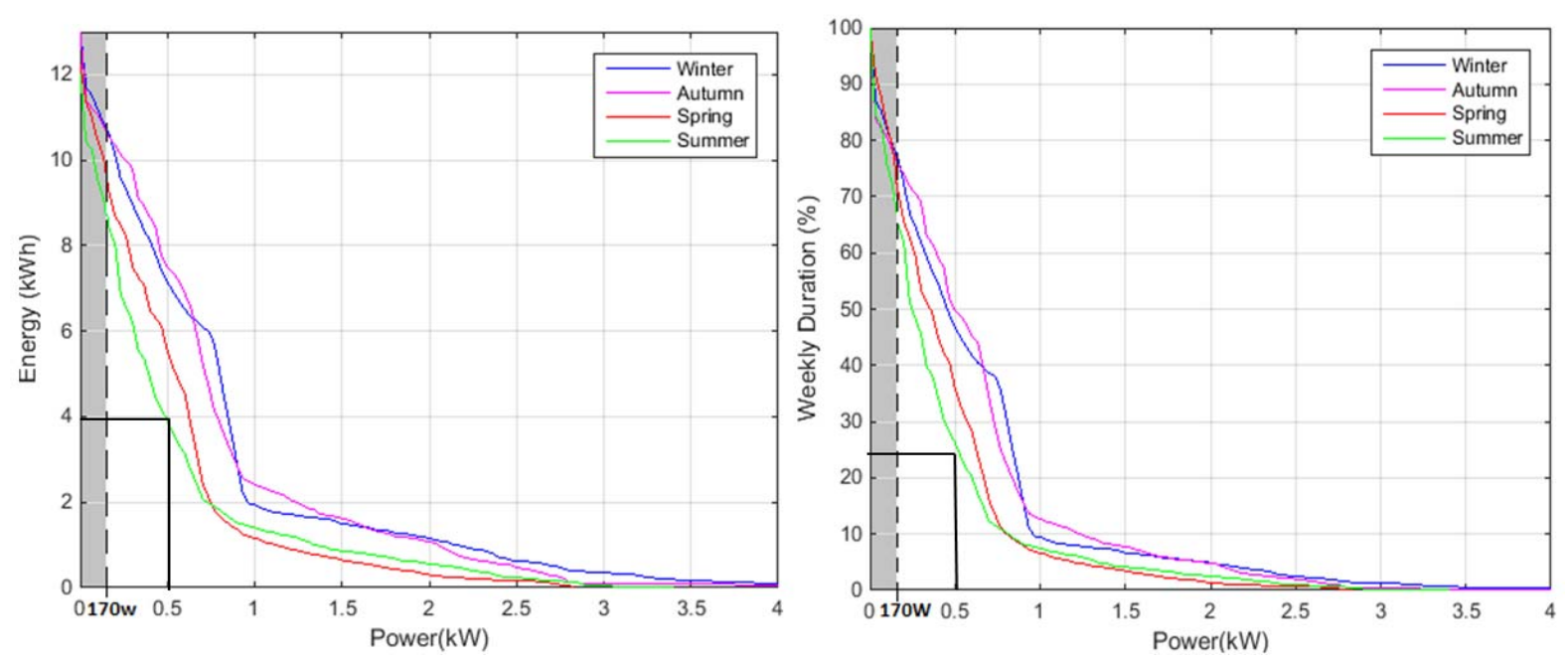

Figs. 2a\&b: Seasonal cumulative converter energy losses (left) and operation battery time (right) for the ESS operational power levels (170W lower cut-off power for the Converter Model 3)

The other approach to preserve the ESS efficiency was to choose the cut-off power so that the one way efficiency is always larger than $70 \%$ which guarantees the roundtrip efficiency is always higher than $50 \%$. Therefore, for the converter model 3 (CM3), the cut-off power was chosen to be $170 \mathrm{~W}$. So, for the CM3, the system shuts down if the required battery power is lower than $170 \mathrm{~W}$, in order to avoid the operation of the ESS at inefficient power levels. As the overnight charging is quite flexible, for the $\mathrm{CM} 3$, if the recharging algorithms would result in overnight charging power lower than $170 \mathrm{~W}$, instead of using all the 7 hours of Economy 7 scheme, the battery is charging for a shorter amount of time but at higher power than the cut-off one. Figs. 3a\&b show the charging pattern and the battery current for a week in summer, for a $4.95 \mathrm{kWh}$ battery. The blue curve illustrates the behaviour of CM1 and the red one of CM3. The light blue shaded areas show the differences during the off-peak overnight charging period; the battery using CM3 charges for a shorter amount of time since the power was below the cutoff one. Moreover, the zoomed areas show that for the CM3, the battery is not operating when the power is below the lower cut-off power threshold.
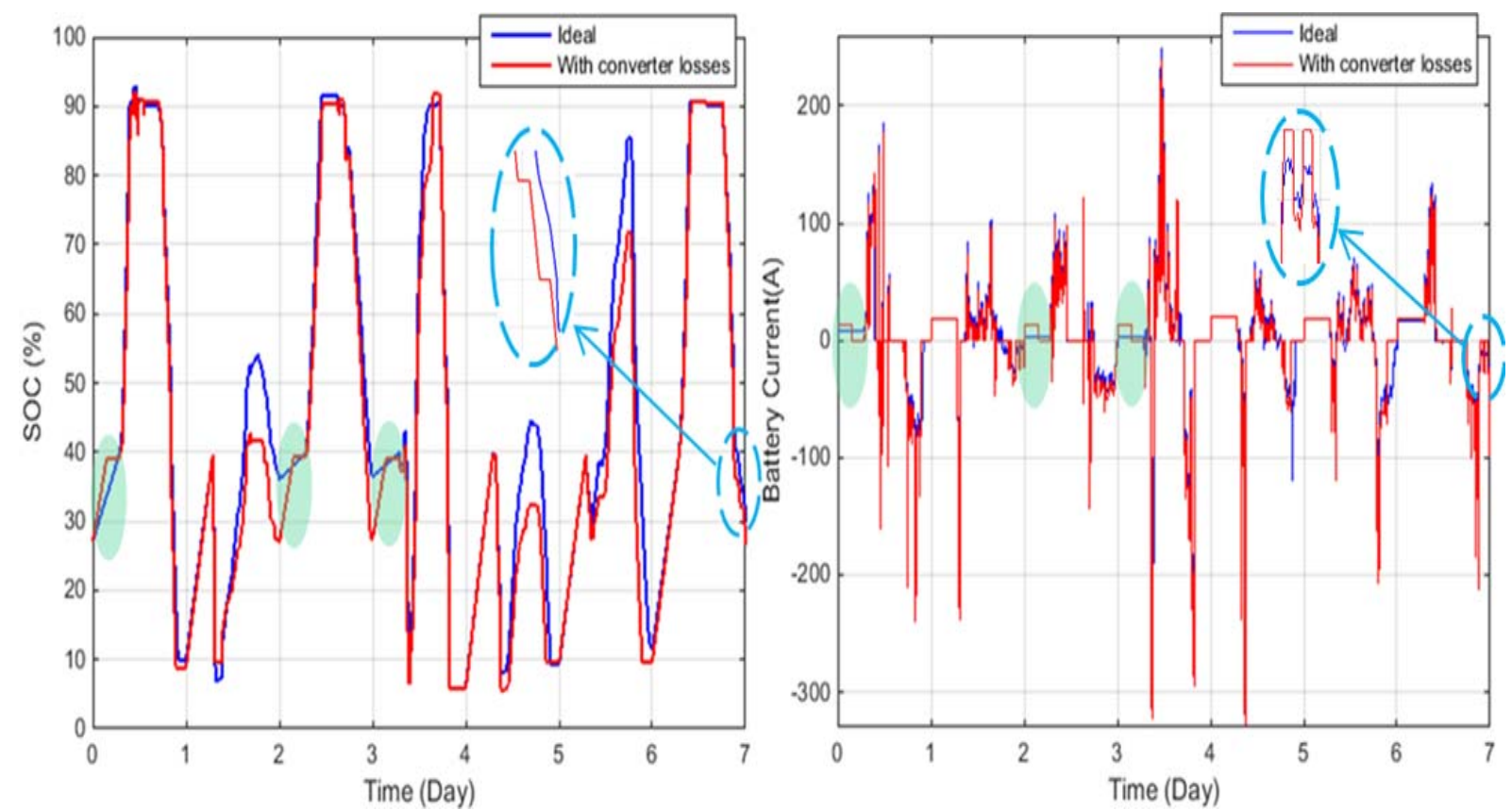

Fig. 3a\&b: Charging pattern and ESS current for Converter Models CM1 (blue) and CM3 (red) for a week in summer (4.95kWh battery -'Constant overnight charging' control algorithm) 


\section{Control Algorithms}

The main goal of the proposed domestic ESS is to reduce the householder's electricity cost to its minimum. This is achieved by using the ESS to capture excess PV generation which otherwise would have been exported (as this export has low revenue for the householder) and also charge the ESS overnight during the off-peak tariff period and discharging during the peak tariff period. The overnight charging level must be chosen correctly: if the battery is not sufficiently charged at night and the day ahead is cloudy, then the battery will probably be fully discharged before the end of the peak period and peak tariff electricity will need to be purchase. On the other hand, if the overnight charge level is too high and the day ahead is sunny, the battery will be fully charged and any excess PV energy must be exported back to the grid. Five different control algorithms were constructed for this work:

1. Constant overnight charging: This is the simplest control approach. The battery charges from 00:00 to 07:00 in order to benefit from Economy 7 pricing scheme, to a preset SOC for every battery size through the whole year. This is the Business as Usual case since there is no access to previous power profiles, or weather forecasts, nor usage of calendar timer. The overnight charging level was chosen to be $0.7,0.5$ and 0.4 (i.e. $70 \%, 50 \%$ and $40 \%$ rated capacity) for three different peak power levels for the PV system installed $-3.5 \mathrm{~kW}, 5.25 \mathrm{~kW}$ and $7.875 \mathrm{~kW}$. These SOC charging levels were chosen since they provide the lowest electricity cost for each PV size. This was determined from simulation studies where the charging levels were successively adjusted between $40 \%$ and $90 \%$ of the battery's nominal capacity, using steps of $10 \%$.

2. Yearly optimized overnight charging: The battery charges overnight to a preset SOC through the year (similar to Control Algorithm 1 - CA1), but in this case, the preset SOC varies depending on the battery capacity. Since for smaller sizes, the capacity is restricted, they discharge quickly, so they must be fully charged overnight, in order to reduce the need to purchase peak tariff electricity during day. On the other hand, for large batteries, the preset SOC must be smaller, since otherwise, it will not be able to capture any excess PV during the day. Additionally, the preset SOC is smaller for higher power PV systems, since the required energy from the power grid is lower.

3. Seasonal optimized overnight charging: The battery charges overnight to a unique charging level which is set for every season. It was assumed that a calendar timer was connected to the meter between the ESS and the power grid in order to change the charging level at the beginning of each season. For summer, the optimal charging level was found to be the minimum one $(0.4-40 \%)$ whereas for winter it was the larger one (0.9-90\%). However, for small batteries, due to their restricted capacity, the most beneficial charging level for all the seasons was found to be 0.9 . Overall, as the battery and PV size increases, the optimal charging level decreases for all seasons.

4. One day before adjusted overnight charging: The overnight level is adjusted by observing the previous day's charging pattern; if during the previous day excess energy was exported to the grid, the overnight charge level for the current day will be decreased by 0.1 (10\% SOC), whereas if peak energy was purchased the day before, the charge level will be increased by 0.1. If the previous day was relatively sunny and the battery was fully charged and had to export excess energy, the overnight level of the following day will be decreased, in order to avoid the need for export again. If during the previous day, peak energy had to be imported, the battery will be charged more the following day. Thus, the previous day's charging pattern is used to tune the preset overnight SOC.

5. Weather predicted overnight charging: In order to implement this algorithm, the system has to have internet access, in order to download the weather forecast for each day. The battery charges overnight up to a preset SOC which leave capacity for the battery to be "topped up" by the predicted PV generation on the following day. With this algorithm, export is eliminated and also, the amount of peak tariff energy purchased is minimum, since the battery is fully charged using all the excess PV energy. For very small battery sizes, the benefits of this algorithm are negligible, since the battery is fully charged overnight. However, for medium sizes, where the need for a welldefined overnight charging level is essential, the usefulness of this scheme is clear. For larger battery sizes, the benefits of this control algorithm are lower, since this algorithm aims to keep the battery fully charged, and it is unable to fully discharge during the evening peak. 


\section{Data Sources}

The data which was used in this study was kindly provided by E.ON [9] and it included the power profile for a UK house with a $3.5 \mathrm{~kW}$ PV system installed. The sample period for the data was 5minutes. The data for one week of winter and summer are shown in Fig. 4. The sample weeks which were examined were: first week of April 2012, fourth week of July 2012, first week of October 2012 and the second week of January 2013. Note that for this study, the losses of the power inverter of the PV were not examined, and it was assumed that they are included to the power generation profile.
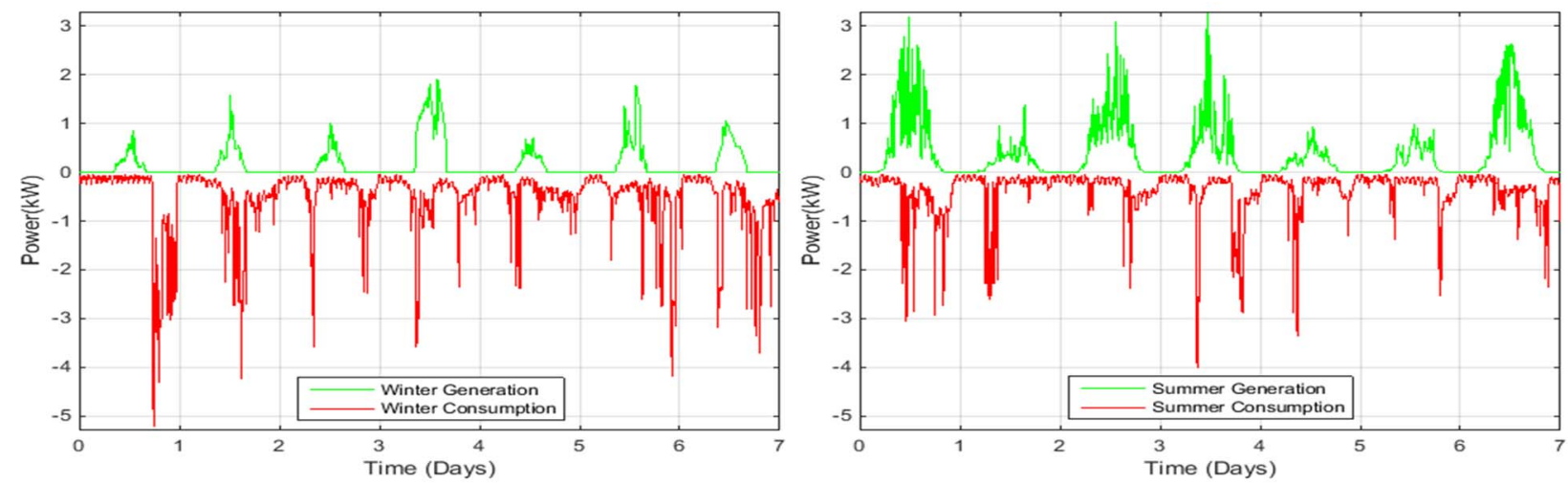

Fig. 4: Power profile data for the household for a week during winter (left) and summer (right) [9]

\section{Results and discussion}

\section{Observation of Charge/Discharge Patterns}

The three different ESS models (100\% efficient power converter (CM1), 95\% efficient converter with (CM2) and without (CM3) lower cut-off threshold) were used for the four seasons, for the five different control algorithms, for different battery capacities and different peak installed PV sizes. Figs. $5 \mathrm{a} \& \mathrm{~b}$ show the charging patterns for CM1, for $9.9 \mathrm{kWh}$ and $4.95 \mathrm{kWh}$ battery capacities for one week in winter and summer. Note that, at the end of each week, the battery must end with the same level of SOC as it started, so a weekly charging cycle is achieved with a fixed amount of energy moving in and out of battery. That is the reason of having different starting SOC for the two seasons.

From Figs. 5a\&b can be seen that both batteries charge overnight to $70 \%$ of their SOC for winter and $40 \%$ for summer (dotted green lines). After 07:00 the battery follows the power profile, until its SOC reaches its limits or till 24:00. The green shaded areas in the figure highlight the differences for different battery sizes. As it can be observed for the larger size, the period when the battery reaches the limits of SOC are shorter than the smaller size. On the other hand, Figs. 6a\&b show the differences in charging patterns when different control algorithms are used. More specifically, they demonstrate the battery capacity for one week during spring and autumn respectively, for a battery of $9.9 \mathrm{kWh}$ for the Control Algorithm 4 (CA4: one day before adjusted overnight charging) and the CA5 (weather predicted overnight charging). For CA4, the initial charging overnight level was chosen to be $60 \%$ of its SOC. Since this algorithm observed the export and the peak purchase energy of the previous day, for the $2^{\text {nd }}$ day of the spring the overnight charging level decreased by $10 \%$, since exports took place on the $1^{\text {st }}$ day, whereas for the week in autumn, it increased by $10 \%$ since peak energy was purchased the previous day. The arrows in Figs6a\&b point to the overnight charging level for each day.

For CA5, from the same figure (Figs.6a\&b) it can be seen that the charging level for each day is not fixed, since it depends on the predicted PV generation. For example, on the $1^{\text {st }}$ day of the week in autumn, the battery is charged to $81 \%$ of its SOC overnight: the battery charged again the following day because due to PV generation reach the higher limit of SOC (90\%). Additionally, in contrast to the $\mathrm{CA} 4$, at the end of the $1^{\text {st }}$ day, the SOC is greater than $10 \%$, and does not need to purchase peak energy from the grid. Furthermore, the $2^{\text {nd }}$ day was relatively cloudy, so the battery charged to its high SOC limit (90\%) in order to avoid purchasing peak electricity at the end of the day. For CA4, peak energy was purchased as shown in the orange shaded areas of Fig.6b. 

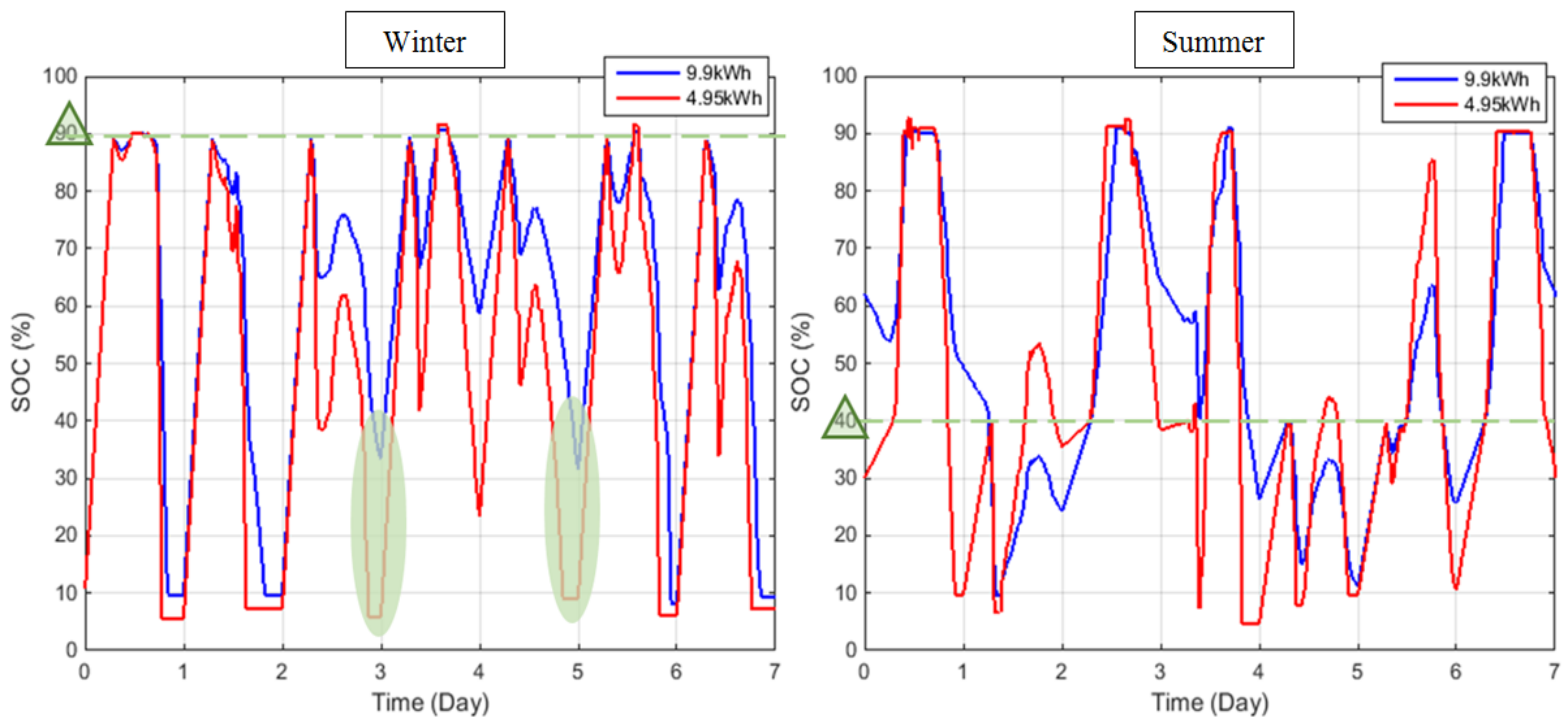

Figs. 5a\&b: Charging patterns for different battery sizes for the constant charging control algorithm
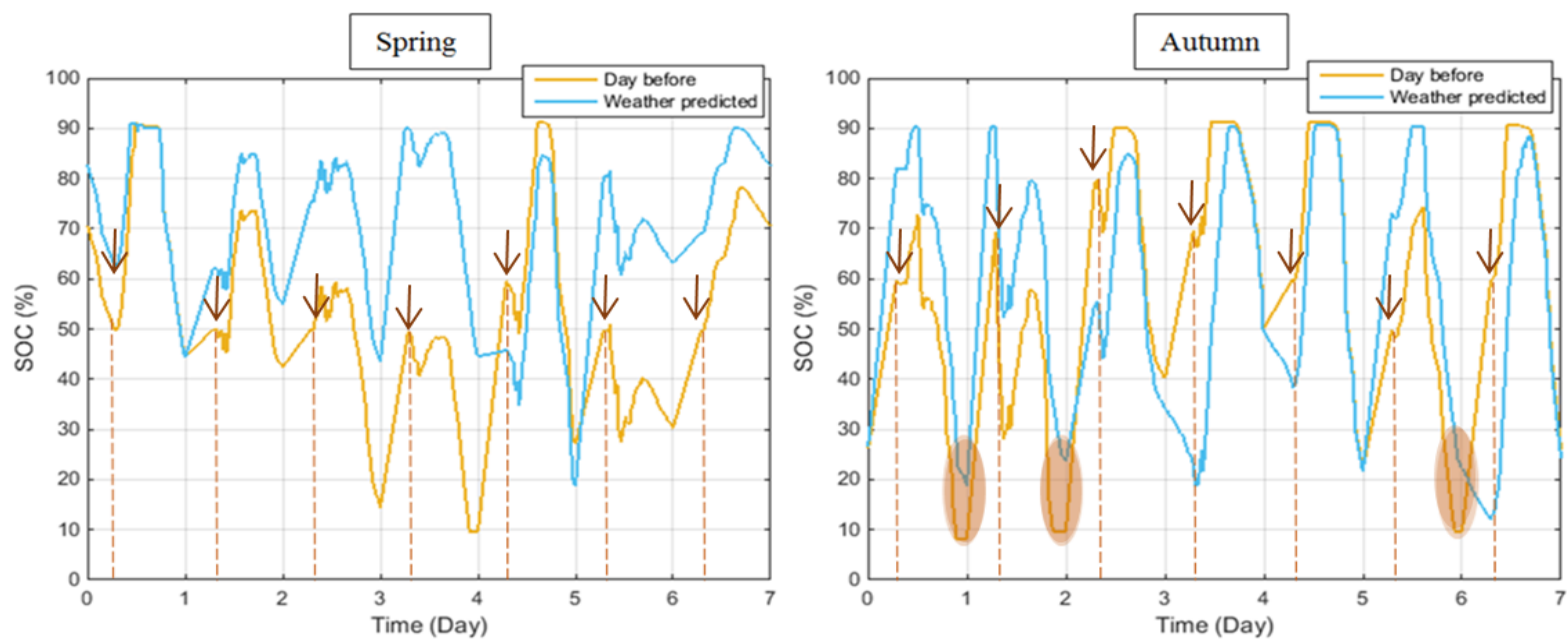

Figs. 6a\&b: Charging patterns for Control Algorithm 4 (Yellow) and Control Algorithm 5 (Blue)

\section{Varying the battery and the PV size}

The five different control algorithms were run for different battery capacities and for three different PV sizes. The original generation data was for an installation of a $3.5 \mathrm{~kW} \mathrm{PV}$, so this was simply scaled by 1.5 and 2.25 , to give $5.25 \mathrm{~kW}$ and a $7.875 \mathrm{~kW}$ PV installed. Three realistic values for UK electricity tariffs were used for this work: the tariff rates were used namely Peak Tariff (07:00 24:00): 15.7p per kWh, Off-Peak Tariff (00:00 - 07:00): 8.4p per kWh and Export Tariff: 4.85p per $\mathrm{kWh}[10]-[12]$.

Fig. 7 summarizes the annual electricity cost for all the five different control algorithms and the three PV sizes as a function of battery capacity for the model with the ideal power converter (CM1). It can be seen that the relationship between the battery size and the annual electricity cost is an exponential decay whose decay is independent of the PV size and the control algorithm. It was anticipated that for very small and very big battery sizes, all the 5 control algorithms would merge and provide roughly the same annual electricity cost. However, for the smaller PV size, the control algorithms' curves do not merge for the large battery capacities. The reason for this is the impact of defining the correct overnight charging level; a wrong choice can lead to either unnecessary export or/and additional peak tariff electricity. Also, as the size of the PV increases, the difference to the electricity cost of the different control algorithms decreases. It can be concluded that, the control algorithms make sense only if the PV size is relatively small for the corresponding consumption profile and the battery size is within the medium size range for the particular installation. 


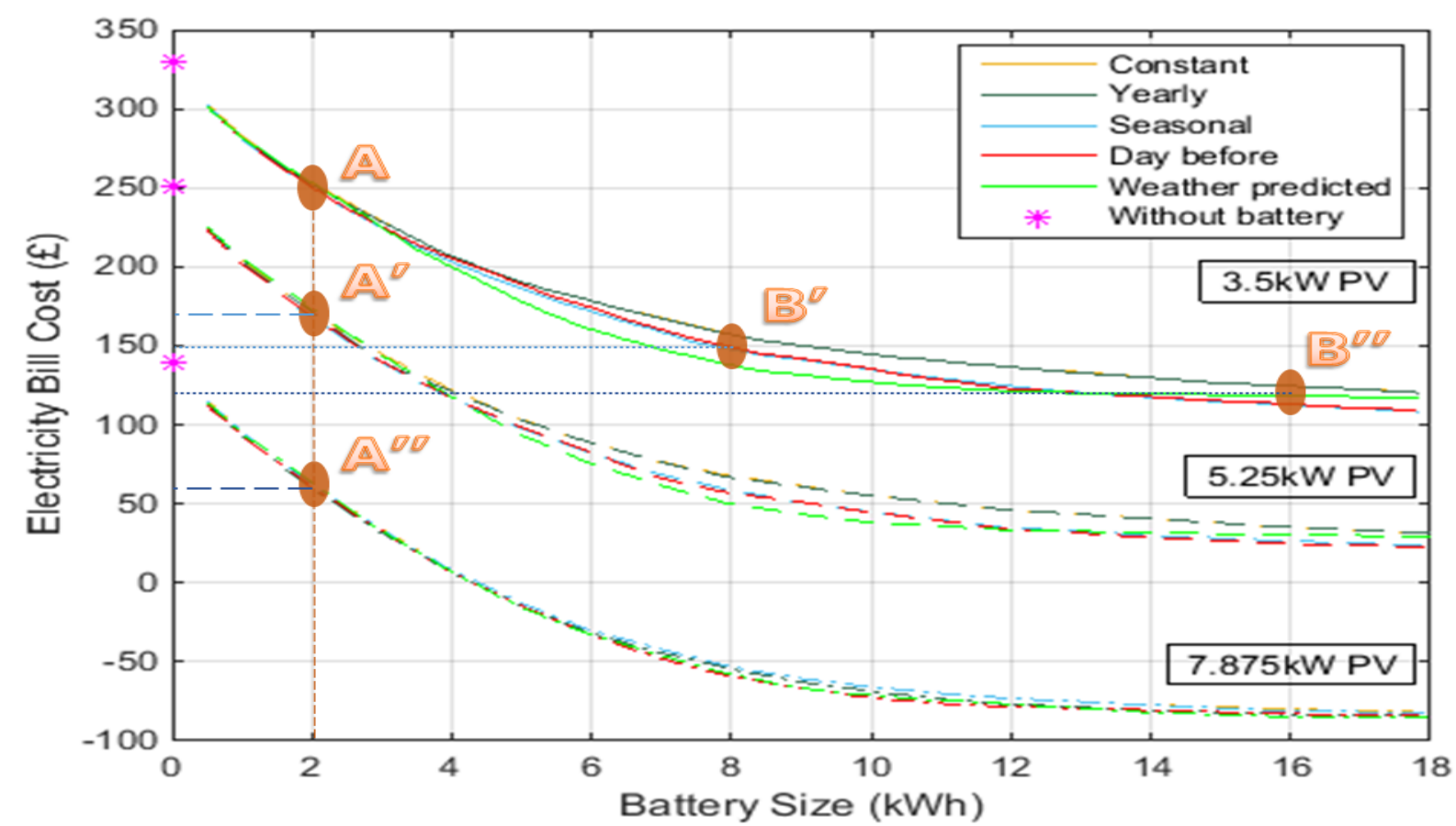

Fig 7: Annual household electricity cost for different control algorithms, battery and PV sizes

In order to identify which is the most suitable battery size in terms of annual electricity cost for a specific domestic application, it has to keep in mind that after a particular battery size, an increase of the battery capacity will not lead to a significant reduction of the annual electricity cost. In order to demonstrate this, two pairs of points were taken from Fig.7. It was assumed that the installation cost remains constant for the PV and battery size considered and that the solar panels cost $£ 0.7$ per Watt, whereas the battery costs $£ 0.2$ per Wh [13]. The pairs of points A', B' and A"' and B"' in Fig.7 have the same investment cost, but they are providing different annual electricity cost to the householders. More specifically by taking the pair A', B', it can be seen that the same reduction in annual electricity cost requires either an increase in 1.5 for the PV or an increase in over 4 times for battery capacity. If the pair A" and B" are examined, an increase of 2.25 in PV power can save almost $£ 50$ on the annual bill (i.e. could be cost effective in the long term), whereas to make the battery 8 times larger is not a cost effective solution in the long term, even though the up-front cost of both solution is the same. Therefore, having a bigger battery is not always the most profitable solution for householders.

\section{Including the power converter losses}

The same analysis as in the previous section was carried out for the two models which include a $95 \%$ efficient converter (CM2 and CM3). The first model considered that the power converter has no lower cut-off limits and so, the ESS can operates over the whole range of power (CM2), whereas for the second model, the ESS shuts down when the operating power was less than the converter's lower cutoff power (CM3). Fig. 8 illustrates the outcomes of the simulations in terms of annual electricity cost for different battery capacities, PV sizes and control algorithms. It can be concluded that the relationship between the annual electricity cost and the battery size does not change by changing the efficiency of the converter. However, the converter's losses increase the annual electricity cost for both PV sizes, by a constant amount. In Fig.8, the light blue shaded areas show the increase in cost between the model with the idea converter and the other two models with the $95 \%$ efficient converter. It can be seen that the difference between the two efficiencies, are more or less the same for all the battery sizes and PVs. Furthermore, the model which operates over the whole power range has a slightly lower electricity cost than the one which does not operate for powers below the lower cut-off. The reason for this is the fact that when the ESS shuts down, the required load during the peak tariff must be covered by the power grid. The savings of converter losses when the ESS is off are less than the cost of purchasing electricity from the grid, and hence, the model which does not operate below the lower cut-off power provides a more expensive electricity bill to the householder. 


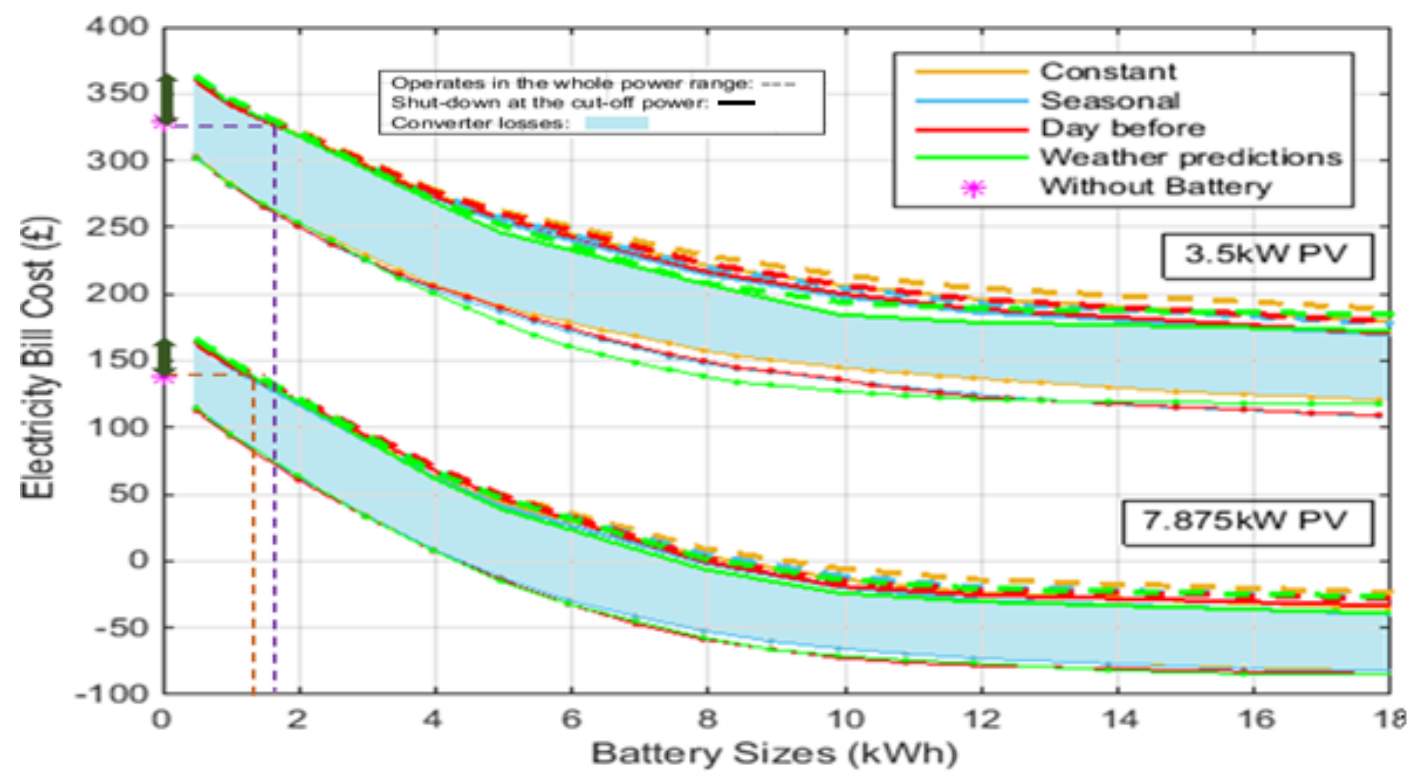

Fig 8: Electricity cost for different control algorithms, battery \& PV sizes (including converter losses)

Moreover, the different control algorithms are distinguished only for medium battery sizes and for the smaller PV, as the CM1. Also, for very small battery sizes, the annual electricity cost is greater than when not using ESS at all. The reason for this is the converter's losses, which add approx. $£ 50$ to the annual electricity cost. So, it can be concluded that if the power converter's losses are taking into account, there is more financial beneficial in not to having ESS installed to the house if the size of the battery is smaller than a particular size (for this application this size was found to be around $2 \mathrm{kWh}$ ).

The losses for the three different models were examined (with and without converter losses). Fig. 9 illustrates the system losses for the three different models - note the different y axes. As was expected, the model which includes the ideal converter has significantly lower losses. What is interesting to observe is that the relationship between losses and battery size is different for the two converter models. For the model with the $100 \%$ efficient converter, the system losses, which include only the ohmic losses of the battery, decrease as the battery size increases. It can be seen that the losses for the CM1 are inversely proportional to the battery size for all the control algorithms and for battery sizes larger than $4 \mathrm{~kW}$. However, the losses sharply decrease for very small batteries despite their very large internal resistance. Nonetheless, for the two models which include the $95 \%$ efficient converter, the losses are increasing as the battery size increases, up to a certain size, and then the system's losses remain constant. Note that CM3 (ESS shuts below lower cut-off power) has lower losses than CM2 (operates over the whole range of power) - the dotted and dashed curves respectively in Fig. 9.

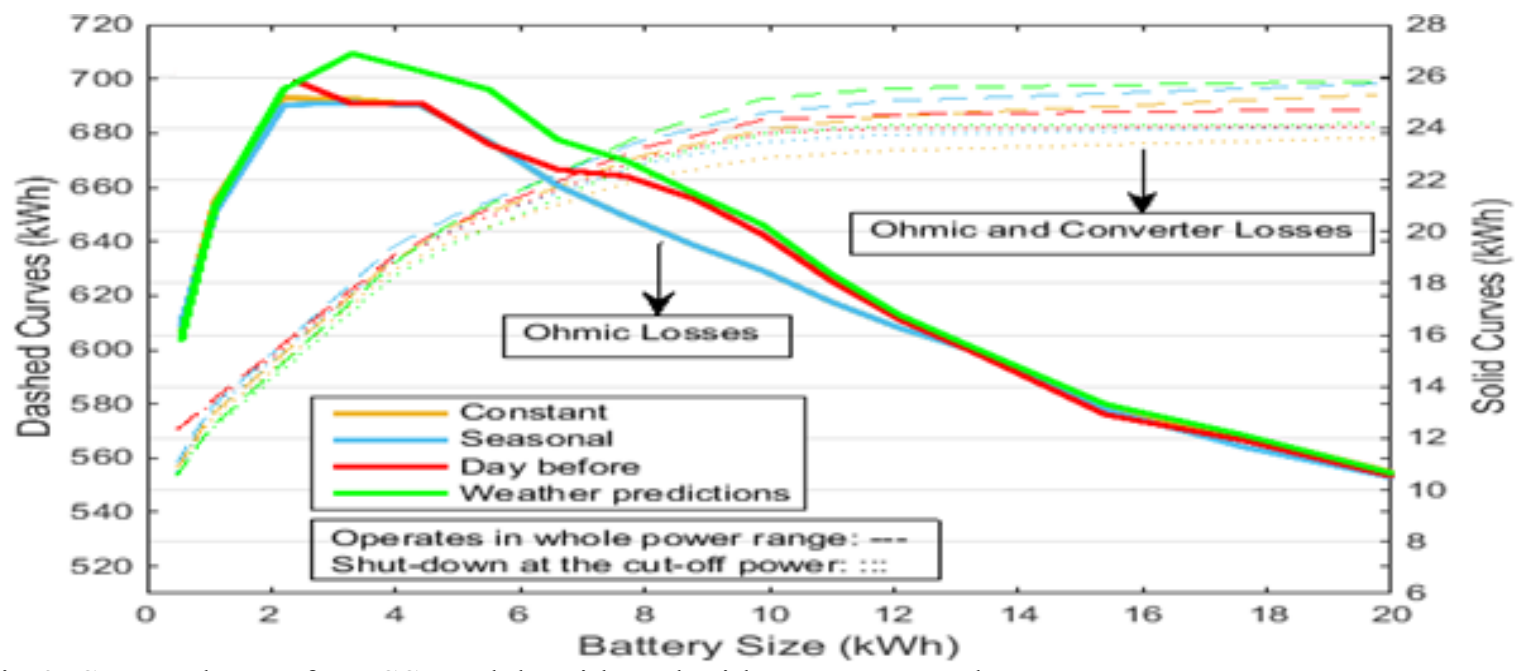

Fig 9: System losses for ESS models with and without converter losses 


\section{Conclusions}

- Technology-based system modelling which can combine the necessary temporal granularity to accurately represent energy storage systems (ESS) is essential.

- The proper function of the ESS models can be demonstrate by observing the charge/discharge patterns over the four seasons for different battery capacities, PV size and control algorithms.

- Five different control algorithms were constructed in order to evaluate their impact on the annual household electricity cost. It was concluded that for all the control algorithms, the relationship between the battery size and the annual electricity cost is an exponential decay whose decay rate is independent of the PV size, the control algorithm used and the efficiency of the converter.

- As the size of the PV increases, the electricity cost difference between the different control algorithms decreases. So, an advance control algorithm makes sense only if the PV is relatively small for the corresponding consumption profile and the battery used is in the medium range of capacity.

- By including the converter losses into the ESS model, the electricity bill cost increased significantly, and for small battery sizes, the bill cost when an ESS is not installed was less than when ESS is used.

- The ohmic losses for battery sizes larger than $4 \mathrm{kWh}$ are inversely proportional to the battery capacity for all the control algorithms. However, the losses are sharply decreased for very small batteries despite their large internal resistance. On the other hand, if the converter's losses are included to the ESS model, the system's losses increase dramatically; as the battery size increases they increase proportionally, up to a certain size, and then the system's losses remain constant for large batteries.

- The model which includes the cut-off power of the converter may have lower system losses but the electricity cost is higher than the one's which operates over the whole range of power, for all the battery capacities and control algorithms. The reason behind this is the fact that the needed demand when the ESS is not operating must be covered by the power grid during the peak tariff period.

- All in all, it can be concluded that the neglect of the power converter's losses can lead to significant errors in analyzing the business case for an ESS, as for this particular study, the power converter's losses increase the annual electricity cost by approx. $17 \%(£ 50)$.

\section{References}

[1] Anuta Oghenetejiri H., et all.: An international review of the implications of regulatory and electricity market structures on the emergence of grid scale electricity storage, Renewable and Sustainable Energy Reviews Journal Vol. 38, pp 489-508, 2014.

[2] Center for Low Carbon Futures: Pathways for energy storage in the UK, 2012.

[3] Rocky Mountain Institute: The economics of battery energy storage. Executive summary, 2015.

[4] Jongerden, M.R. \& Haverkort, B.R.: Battery Modelling, Available Online, 2015.

[5] Shuhui, Li. Bao, Ke.: Study of battery modeling using mathematical and circuit oriented approaches. 2011 IEEE Power and Energy Society General Meeting., paper 12303277.

[6]Robyn A, Jackey.: A Simple, Effective Lead-Acid Battery Modeling Process for Electrical System Component Selection. The Mathworks, Inc.,2007.

[7] Leoch Company: OCV VS Capacity, Datasheet for a 12V-33Ah deep cycle Lead-Acid Battery.

[8] Divya K.C., Ostergaard Jacob : Battery energy storage technology for power systems-An overview, Electric Power SystemsResearch Journal, Vol. 79, pp 511-520, 2009.

[9] E.ON. (2013) " Representative household consumption and PV generation data, Thinking Energy program".

[10] ofgem: Feed-in Tariffs: get money for generating your own electricity, 2015.

[11] Department of Energy \& Climate Change: UK National Energy Efficiency Action Plan, 2014.

[12] Energy Saving Trust: Fuel prices and carbon intensity, 2015.

[13] CompareMySolar Company: Solar panel prices: Compare prices for local installers, Available Online, 2016. 